Meta

Journal des traducteurs

Translators' Journal

\title{
Quelques aspects du métier de terminologue
}

\section{Gabriel Otman}

Volume 35, numéro 4, décembre 1990

URI : https://id.erudit.org/iderudit/003771ar

DOI : https://doi.org/10.7202/003771ar

Aller au sommaire du numéro

Éditeur(s)

Les Presses de l'Université de Montréal

ISSN

0026-0452 (imprimé)

1492-1421 (numérique)

Découvrir la revue

Citer cet article

Otman, G. (1990). Quelques aspects du métier de terminologue. Meta, 35(4),

759-768. https://doi.org/10.7202/003771ar d'utilisation que vous pouvez consulter en ligne.

https://apropos.erudit.org/fr/usagers/politique-dutilisation/ 


\section{QUELQUES ASPECTS DU MÉTIER DE TERMINOLOGUE}

\section{INTRODUCTION}

Sur le plan scientifique, la terminologie commence à s'imposer en tant que discipline autonome dans le champ de la lexicologie. Sur le plan institutionnel, elle est perçue comme un maillon nécessaire à la cohérence d'une politique linguistique. Sur le plan économique, elle se crée progressivement une place dans les entreprises privées au sein des services de communication : traduction, documentation, mercatique.

La profession de terminologue, quant à elle, n'a pas encore d'existence institutionnelle, en France en tout cas, puisque, d'une part, l'ONISEP (Office National d'Information sur les Enseignements et les Professions) ne répertorie pas encore cette activité dans ses listes de professions nouvelles et que, d'autre part, les entreprises employant des terminologues utilisent des appellations sous-entendant des polyvalences du type : terminologue-traducteur, terminologue-informaticien... Il n'en demeure pas moins que la fonction existe, que de nombreuses tâches lui sont affectées, et que cette situation de fait ne peut qu'aboutir à une stabilisation de la fonction et à une définition de ses statuts; surtout si, en amont, les structures de formation en terminologie, universitaires et professionnelles, se développent parallèlement, avec, en particulier, la création de diplômes de terminologie.

Au Canada déjà, l'administration reconnaît officiellement la fonction de terminologue (Moosbrugger, 1988), et dans divers pays comme la Suisse ou le Luxembourg, les organismes internationaux disposent de banques de données terminologiques intégrées à des services de terminologie pourvus de personnel spécialisé.

\section{LA TERMINOLOGIE D'ENTREPRISE}

Le service terminologique - dans les entreprises qui ont jugé utile de s'en doter (voir l'exemple de ASCOM HASLER SA dans Neuhaus (1988)) — se trouve placé à un carrefour stratégique d'échanges entre offres et demandes de services. Physiquement, on a spontanément rattaché le service terminologique au bureau des traducteurs. Pratiquement, il ne peut se passer des services informatiques et télématiques ainsi que des services de documentation de l'entreprise. Stratégiquement, le service de terminologie collabore avec les services d'information et de publicité, de marketing et de vente d'une part, et les départements de recherche et développement d'autre part (voir schéma cidessous). 


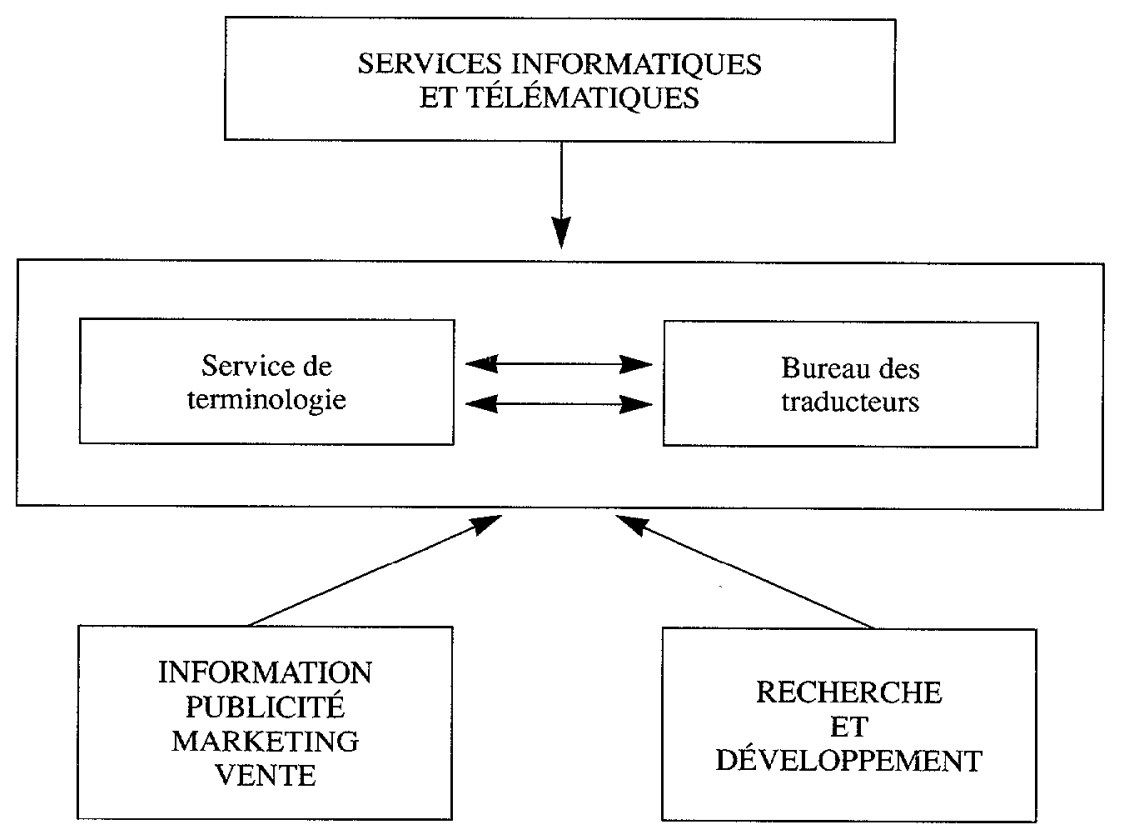

Schéma 1

Place du service de terminologie au sein d'une entreprise

\section{LES TÂCHES DU TERMINOLOGUE}

Si le terminologue a besoin de connaître parfaitement les rouages de l'entreprise à laquelle il collabore, c'est parce que, dans la pratique, tous les décideurs de cette entreprise et, en particulier, ceux des départements mentionnés dans le schéma ci-dessus peuvent être amenés à solliciter des services ponctuels de terminologie ; par exemple :

- trouver l'équivalent français d'un terme technique étranger,

- choisir l'appellation d'un produit nouveau,

- donner un avis sur la formulation de slogans publicitaires,

- uniformiser les dénominations de pièces, de produits ou même d'unités administratives,

- rédiger divers documents ou revoir la rédaction de documents techniques émanant de certains services... (Cartier, 1985).

La fonction essentielle du terminologue est d'assister le traducteur. Rien d'étonnant donc au fait que l'on trouve tant de doubles casquettes «traducteur-terminologue», les terminologues «spécialisés» n'étant bien souvent, en réalité, que d'anciens traducteurs chevronnés. Cette expérience préalable de la traduction est même jugée essentielle (Hamilton, 1988) dans la mesure où l'on estime que l'activité terminologique doit être perçue sous l'angle de l'assistance à la traduction (augmentation de la qualité des traductions et du rendement des traducteurs, développement de systèmes de traduction assistée par ordinateur...). 
Le terminologue dresse, puis met à jour (et vérifie) des vocabulaires techniques propres aux activités de l'entreprise. Ce travail de longue haleine est effectué à partir de la documentation technique interne fournie par les services spécialisés sans omettre le recours aux documents externes, cela afin d'éviter la création d'une terminologie d'entreprise divergente de la terminologie courante (ou parfois pour encourager la création d'une telle terminologie pour des raisons de marketing par exemple).

Le terminologue remplit souvent les fonctions de «vérificateur-relecteur» de traductions. Cette relecture pourra également être l'occasion de mises à jour de fiches terminologiques ou de la découverte de nouveaux ensembles de termes à étudier. Le traducteur-spécialiste d'un domaine, qui possède souvent par-devers lui des glossaires personnels sur fiches cartonnées accumulées au fil des années et de l'expérience, détient là une matière première inestimable en vue d'un «raffinage» terminologique. A priori, on pourrait estimer, pour ces raisons, qu'il est le plus apte à jouer le rôle de terminographeexpert. En fait, une démarche systématique de ce type entraînerait un essaimage néfaste de l'activité terminologique pour aboutir à la création de «parcelles» de terminologie non normalisées, dont le «remembrement» nécessiterait un surcroît de travail peu économique.

Par contre, le terminologue professionnel, doué d'un savoir théorique et d'un savoir-faire terminologique doublés d'une bonne connaissance des exigences de la traduction technique, saura être le maître d'œuvre d'un projet cohérent de réalisation de bases de données terminologiques multilingues. Cette compétence inclut la maîtrise d'outils informatiques avancés (système de gestion de bases de données, stockage et transmission de données sur disque optique ou par voie télématique, système de TAO...).

\section{ACTIVITÉS PONCTUELLES ET THÉMATIQUES DU TERMINOLOGUE}

D'après un sondage réalisé par la Société des traducteurs du Québec (STQ) sur l'évaluation du rendement des terminologues, et dont les résultats ont été publiés en mars 1988 (voir extraits en annexe 1), le terminologue moyen partage son temps de travail quotidien à parts égales (environ quatre heures) entre activités «ponctuelles» et activités «thématiques».

\subsection{ACTIVITÉS PONCTUELLES}

Le terminologue est une personne qui doit être disponible à tout moment: pour assister les traducteurs, les documentalistes, les spécialistes internes ou pour répondre à diverses demandes ponctuelles émanant de services externes. Cette activité de «recours permanent», de «ligne ouverte» est surtout l'apanage des terminologues implantés dans des institutions publiques parce qu'ils sont considérés par leur clientèle comme des références fiables, d'une part, et des sources d'informations généralement gratuites, d'autre part.

D'après l'enquête citée, un terminologue répond en moyenne à une dizaine de demandes ponctuelles par jour ouvrable et il consacre à chacune d'elles une moyenne de 23 minutes.

\subsection{ACTIVITÉS THÉMATIQUES}

Il s'agit principalement de la réalisation de vocabulaires monolingues, bilingues ou multilingues. Ce travail peut se décomposer en huit phases:
i) - recherche documentaire
ii) - dépouillement
iii) - rédaction de fiches
iv) - consultation de spécialistes
v) - révision/correction
vi) - publication/diffusion 
vii) - informatisation/mise sur serveur ou base de données terminologiques

viii) - mise à jour/entretien/ dépoussiérage

dont les quatre premières peuvent être menées de front. Détaillons-les:

\subsubsection{RECHERCHE DOCUMENTAIRE}

La moitié des services de terminologie seulement dispose d'une assistance documentaire; ce qui revient à dire que le terminologue reste encore généralement son propre documentaliste et c'est à lui qu'incombent les tâches de repérage, d'acquisition et d'indexation de ses documents.

\subsubsection{DÉPOUILLEMENT}

Selon les besoins, le terminologue optera pour le dépouillement de textes de spécialité et/ou de traductions. L'activité de dépouillement, très gourmande en temps, devrait pouvoir être au moins partiellement automatisée (voir à ce sujet les travaux du centre ATO (analyse de texte par ordinateur) de l'Université du Québec à Montréal sur le développement d'un système de dépouillement terminologique assisté par ordinateur).

\subsubsection{RÉDACTION DE FICHES}

Le temps consacré à la rédaction de fiches dépend largement de l'extension de celles-ci. Une fiche terminologique «de traducteur» comportant classiquement quatre à cinq champs (vedette, domaine, définition, équivalents en $\mathbf{n}$ langues) pourra être remplie en quelques minutes ( $7 \mathrm{mn}$ en moyenne d'après l'enquête citée) alors que la rédaction d'une fiche complète (plus de dix champs) peut prendre au terminologue plus d'une demi-heure de son temps. La fiche type du Centre de terminologie et de néologie comportant plus de 25 champs selon les usages - (voir modèle pour la terminologie de l'intelligence artificielle en annexe 2), particulièrement détaillée et riche en informations sémantiques (champs <ACTION TYPIQUE $><$ OBJET CONNEXE $><$ SORTE DE $>$ $<$ PARTIE DE $><$ ANAPHORE $><$ CONSTITUANTS $>$... ) et syntaxiques $<$ COMBINAISON> <PARTIE DU DISCOURS>, est un exemple de fiche extensive à finalité terminologique et lexicographique qui exige un temps de rédaction non négligeable. Pour une description détaillée de cette fiche, nous renvoyons le lecteur à (Lerat, 1988).

\subsubsection{CONSULTATION DE SPÉCIALISTES}

Le terminologue d'entreprise n'a, en principe, aucune peine à recueillir des renseignements terminologiques de qualité à l'interne, auprès des spécialistes maison (ingénieurs, techniciens, rédacteurs techniques...) maniant parfaitement le vocabulaire de leur spécialité. Cette tâche de consultation-échange a un double but: valider le travail terminologique et normaliser la terminologie à répandre à l'intérieur comme à l'extérieur de l'entreprise (rédaction des modes d'emploi, des notices d'utilisateur, des manuels techniques, des nomenclatures de pièces détachées...) (Neuhaus, 1988).

Des consultations de ce type, qui ne doivent pas se limiter à des échanges à sens unique, se font aussi vers l'extérieur de l'entreprise. Le terminologue a alors pour vocation d'être un créateur de réseaux de contacts entre experts d'un même domaine technique. Le fruit de ces investigations auprès de spécialistes donnera lieu à des révisions et à des corrections de fiches.

\section{LES PRODUITS TERMINOLOGIQUES}

Bélanger (1985) distingue les produits primaires: — la fiche terminologique, l'étude terminologique, la chronique de langue et les banques de terminologie - des produits dérivés: - les formulaires, les catalogues, les notices techniques, les documents audiovisuels et les systèmes informatiques de gestion (mots clés, index...). 
Ce sont les produits dérivés qui, d'après Nycole Bélanger, assurent la diffusion réelle des terminologies bien plus efficacement que les produits primaires. Le terminologue doit être conscient de cet état de fait et donc ne pas porter tout son effort sur la seule réalisation de fichiers terminologiques.

\section{INFORMATISATION DU SERVICE TERMINOLOGIQUE}

Le service terminologique n'a de raison d'être que si ses productions trouvent un usage effectif auprès des utilisateurs. À l'intérieur d'une entreprise dotée d'un réseau informatique, la banque de données terminologiques doit pouvoir être consultée directement par tout le personnel concerné à partir de leur station de travail individuelle.

Il ressort de l'enquête de la STQ que seulement $39 \%$ des fichiers de terminologie sont déjà informatisés et $52 \%$ en voie d'informatisation. Ces chiffres peuvent paraître décevants. Par contre, et cette nouvelle est rassurante pour l'avenir, $64 \%$ des terminologues participent activement à l'informatisation de leur service (analyse des besoins, choix des matériels et logiciels, évaluation des coûts, modalités de rattachement et d'échanges avec les grandes banques de données, etc.).

\section{LES CONNAISSANCES ET COMPÉTENCES REQUISES DU TERMINOLOGUE}

L'inventaire non exhaustif que nous venons de dresser aura permis de montrer la variété des tâches et des activités du terminologue. Cette diversité sous-entend que ce dernier possède à la fois une vaste culture générale et des connaissances spécialisées approfondies, doublées de la pratique d'une ou de plusieurs langues étrangères sans oublier, bien entendu, une formation théorique en linguistique générale, lexicologie et terminologie ainsi qu'une initiation aux techniques documentaires.

Toutes ces connaissances exigent, de plus, d'être régulièrement renouvelées et complétées. La curiosité intellectuelle, l'ouverture d'esprit semblent être les qualités principales requises pour réussir en terminologie. Le terminologue étant également considéré comme un communicateur, il est bon qu'il possède le sens des relations humaines et du contact.

Une formation strictement limitée aux méthodologies terminologiques (élaboration de fiches, arborescence de domaines, gestion de bases de données terminologiques, recherche documentaire...) est insuffisante pour remplir l'ensemble des tâches qu'exige le métier de terminologue en entreprise. Toutefois, cette polyvalence, nécessaire en milieu professionnel, ne peut être entièrement transmise dans le cadre universitaire si cette institution ne fait pas l'effort d'inclure dans son cycle de formation des stages en entreprise dans des services de terminologie (ou des services de traduction dans la mesure où les premiers ne sont pas encore suffisamment nombreux pour accueillir tous les étudiants en formation). Cette «intrusion» de spécialistes en terminologie dans des centres de traduction aura indubitablement des effets bénéfiques sur la création de postes de terminologues car «l'employeur arrive seulement à reconnaître les avantages d'une innovation lorsqu'il en voit les résultats concrets» (Shipton, 1988).

Moosbrugger (1988), outre les spécialisations en traduction et documentation, mentionne un troisième volet indispensable à la formation en terminologie: l'informatique. La discipline est, bien évidemment, trop vaste et trop technique pour être totalement assimilée par des non-spécialistes. Il faut donc se satisfaire d'une tranche spécifique de l'informatique : l' informatique appliquée à la terminologie.

\section{MISSIONS ET COLLOQUES}

La réflexion théorique et appliquée en terminologie est féconde; cette abondance se traduit par l'organisation régulière de colloques, de symposiums, de tables rondes 
consacrés à la terminologie, à ses applications, à ses débouchés professionnels aussi bien en Europe qu'outre-Atlantique. Ces rencontres qui mêlent théoriciens, praticiens, industriels et responsables politiques sont un complément indispensable à la formation continue.

L'enquête précitée montre qu'à l'heure actuelle les terminologues en activité ne consacrent que $10 \%$ de leur temps à la poursuite de leur formation, et accordent quatre journées annuellement à des colloques. Par ailleurs, il semble souhaitable que le terminologue ait la possibilité de fréquenter des salons et des expositions dédiés à l'informatique, à la bureautique, aux systèmes de documentation électroniques, et d'assister à des rencontres traitant de traduction assistée par ordinateur auxquelles il faut aujourd'hui ajouter les manifestations consacrées à l'intelligence artificielle (en particulier si elles touchent à des questions portant sur le traitement du langage naturel, la linguistique computationnelle et la communication homme-machine).

\section{PERSPECTIVES D'AVENIR}

Selon Rey (1985), «non seulement la terminologie n'intervient que lorsqu'il y a enjeu historique, politique ou économique, mais elle ne peut agir qu'au sein de systèmes complexes de communication où les images et d'autres sons que ceux du langage sont impliqués».

Si le métier de terminologue vient de sortir de sa période de balbutiement, il n'a pas encore atteint sa totale maturité : adaptation au marché de l'emploi, formation adéquate, intégration dans les structures existantes et prise en compte de la dimension politicoéconomique de la fonction.

NOTE : Cet article reproduit la communication de l'auteur présentée à l'Université de Rouen le 19 avril 1989.

\section{BIBLIOGRAPHIE}

BÉLANGER, Nycole (1985): «La diversité des produits terminologiques», Actes du sixième colloque OLF$S T Q$ de terminologie, novembre 1985, Société des traducteurs du Québec, pp. 135-145.

CARTIER, Nathalie (1985) : «L'activité terminologique dans l'entreprise privée», TermNet News, 9, pp. 31-35.

HAMILTON, Ian (1988): «The profession of terminologist in the United Nations Office at Geneva», Actes de «Rencontre internationale sur l'enseignement de la terminologie», 21-22 septembre 1988, Université de Genève, pp. 158-159.

LERAT, Pierre (1988): «Terminologie et sémantique descriptive», La Banque des mots, 36, $4^{\mathrm{e}}$ trimestre 1988, pp. 11-30.

MOOSBRUGGER, Mireille (1988) : «Marché du travail, besoins des employeurs, formation préalable, formation continue, terminologues professionnels ou simples utilisateurs...», Parallèles, 10, Université de Genève, pp. 53-54.

NEUHAUS, Jean-Pierre (1988): «Le terminologue au sein d'une entreprise privée», Actes de «Rencontre internationale sur l' enseignement de la terminologie», Université de Genève, septembre 1988, pp. 195-208.

REY, Alain (1985) : «Les fonctions du terminologue», Actes du sixième colloque OLF-STQ de terminologie, novembre 1985, Société des traducteurs, Québec, pp. 87-108.

SHIPTON, Janine (1988): «L'industrie suisse a-t-elle besoin de terminologues?», Actes de «Rencontre internationale sur l'enseignement de la terminologie», septembre 1988, Université de Genève, pp. 181-194.

STQ (1988) : «Sondage sur l'évaluation du rendement des terminologues: analyse des résultats», Société des traducteurs du Québec, Montréal, mars 1988, 36 pages, multigraphié. 
Annexe 1

SONDAGE SUR L'ÉVALUATION DU RENDEMENT DES TERMINOLOGUES (mars 1988)

(Société des traducteurs du Québec)

(extraits)

RECHERCHE THÉMATIQUE

Établissez-vous des lexiques?

- oui $(74 \%)$

Combien de temps consacrez-vous en moyenne à la production d'un lexique de 100 termes selon les méthodes terminologiques reconnues?

- réponse moyenne pondérée : 86 jours

Quel pourcentage de ce temps consacrez-vous à la recherche documentaire?

- rmp : $14 \%$ soit 12 jours

Quel pourcentage de ce temps consacrez-vous à la consultation de spécialistes?

- rmp : $11 \%$ soit 9 jours

Quel pourcentage de ce temps consacre un spécialiste collaborant à la réalisation d'un tel lexique ?

- rmp : $8 \%$ soit 6 jours

Quel pourcentage de ce temps est consacré à la révision par le réviseur?

armp : $11 \%$ soit 9 jours

Dans quelle proportion les recherches thématiques donnent-elles lieu à des mises à jour de fiches?

- $\operatorname{rmp}: 1 \operatorname{sur} 7$

Combien de temps consacrez-vous en moyenne à la mise à jour d'une fiche?

- rmp: 16 minutes

\section{RECHERCHE PONCTUELLE}

Combien de demandes ponctuelles traitez-vous en moyenne par jour (rédaction de fiches exclue)?

- rmp : 10 demandes

Combien de temps consacrez-vous en moyenne à une demande ponctuelle ?

- rmp : 23 minutes

Dans quelle proportion les demandes ponctuelles donnent-elles lieu à des mises à jour de fiches ?

- $\mathrm{rmp}: 1$ sur 10

Combien de temps consacrez-vous en moyenne à la mise à jour d'une fiche?

- rmp: 14 minutes

DÉPOUILLEMENT TERMINOLOGIQUE

Faites-vous du dépouillement de traductions?

m oui : $51,7 \%$

Combien d'heures y consacrez-vous en moyenne par mois ?

- rmp : 6 heures

Combien de temps consacrez-vous en moyenne à l'établissement d'une fiche bilingue issue de ce dépouillement?

- rmp: 11 minutes

Faites-vous du dépouillement terminologique de textes spécialisés (ex. : articles de périodiques) ?

oui : $64,8 \%$

Quel temps y consacrez-vous en moyenne par mois?

- rmp: 6 heures 
Combien de termes recueillez-vous par mois?

- rmp : 25 termes

\section{RÉDACTION DE FICHES}

Combien de temps consacrez-vous en moyenne à la rédaction d'une fiche simple (vedette, équivalent, sources et domaines)?

- mp: 7 minutes

Combien de temps consacrez-vous en moyenne à la rédaction et à la relecture d'une fiche complète (vedette, equivalent, note, contexte, définition, domaines, etc.) ?

- rmp : 32 minutes

\section{RÉDACTION}

Faites-vous de la rédaction de bulletins de terminologie composés d'articles, d'études et de chroniques de langue?

- oui : $57,1 \%$

Combien de temps consacrez-vous en moyenne à la rédaction d'un bulletin de terminologie de 2 pages du type suivant:

article : rmp : 3 jours

- étude : rmp : 3 jours

- chronique de langue : $\mathrm{mp}: 7$ heures

Combien en rédigez-vous par année?

- article: $\mathrm{rmp}: 2$

- études : rmp : 2

- chroniques de langue : rmp : 3

\section{PRODUCTION DE PUBLICATIONS}

Combien de temps consacrez-vous en moyenne à la préparation du manuscrit d'un ouvrage terminologique de 100 termes (illustrations, rédaction des pages liminaires et de la bibliographie, élaboration et inversion, mise en forme et relecture)?

- rmp : 3 semaines

Combien de temps consacrez-vous en moyenne à la préparation du manuscrit d'un bulletin de terminologie de 2 pages composé d'articles, d'études et de chroniques de langue?

- mop : 1 jour

Vos publications font-elles l'objet d'un traitement graphique (illustrations, maquette, typographie, choix de caractères et de papier, montage, etc.) par un service spécialisé ?

- oui : $65,7 \%$

Si vous vous en chargez vous-même, combien de temps consacrez-vous au traitement graphique du manuscrit d'un ouvrage terminologique de 100 termes?

- sans illustrations : rmp : 3 jours

- avec illustrations : rmp : 8 jours

Disposez-vous des services d'un correcteur d'épreuves?

- non : $69,5 \%$

Si non, combien de temps consacrez-vous en moyenne à la correction d'épreuves du manuscrit d'un ouvrage terminologique de 100 termes?

- $\mathrm{rmp}: 4$ jours

Combien de temps consacrez-yous en moyenne à l'édition du manuscrit d'un bulletin de terminologie de 2 pages composé d'articles, d'études et de chroniques de langue ?

- rmp: 1 jour

\section{DIFFUSION DE PUBLICATIONS}

Disposez-vous d'un service de diffusion?

- non : $54,8 \%$ 
Si oui, combien de temps consacrez-vous vous-même en moyenne par mois à la stratégie de diffusion d'une publication (choix des destinataires, lettre publicitaire, chronique dans le journal de l'entreprise, etc.)?

- $\operatorname{rmp}: 2$ jours

\section{DOCUMENTATION}

Avez-vous une documentaliste à votre service?

a oui : $51,5 \%$

Faites-vous vous-même de la recherche documentaire pour les clients (traducteurs, spécialistes, etc.) ?

aui : $57,6 \%$

Faites-vous vous-même du repérage de documentation dans des revues techniques, des catalogues d'éditeurs, etc. ?

- oui : $74,2 \%$

Faites-vous vous-même l'indexation d'ouvrages et d'articles de périodiques spécialisés ?

non : $68,2 \%$

Combien de temps consacrez-vous en moyenne par mois aux activités précédentes?

- $\operatorname{rmp}: 3$ heures

Faites-vous de l'information documentaire?

- oui : $54,7 \%$

Combien de temps consacrez-vous en moyenne par mois à l'information documentaire?

- $\operatorname{rmp}: 2$ heures

Combien de temps consacrez-vous en moyenne par mois à d'autres activités reliées à la documentation (gestion, budget, correspondance, commandes, épuration de dossiers documentaires, etc.)?

- $\operatorname{rmp}: 3$ heures

\section{INFORMATISATION ET GESTION DES FICHIERS}

Votre fichier terminologique

- est-il en voie d'informatisation : - oui : $52,4 \%$

- est-il informatisé : - non : $61,2 \%$

Participez-vous à l'informatisation de vos activités?

- oui : $63,8 \%$

Si oui, combien de temps consacrez-vous en moyenne par mois à l'informatisation (analyse des besoins, échanges avec spécialistes, analyse de matériels et de logiciels, évaluation des coûts, etc.)?

- $\operatorname{rmp}: 10$ heures

Combien de temps consacrez-vous en moyenne par mois à vos relations avec les banques de terminologie (rattachement, consultation, envoi de fiches, etc.) ?

- $\operatorname{rmp}: 6$ heures

Faites-vous de la mise à jour (modification et annulation) de fiches terminologiques?

- oui : $83,9 \%$

Combien de temps consacrez-vous en moyenne par mois à la mise à jour de fiches?

- $\operatorname{rmp}: 10$ heures

\section{AUTRES ACTIVITÉS}

Quel pourcentage de votre temps consacrez-vous en moyenne à la gestion (personnel et budget, planification de projets, etc.)?

1. $\operatorname{mip}: 31 \%$

Quel pourcentage de votre temps consacrez-vous en moyenne aux relations avec les services et organismes de terminologie (expertise de publications, enquêtes, etc.)?

1. $\operatorname{rmp}: 6 \%$ 
Quel pourcentage de votre temps consacrez-vous en moyenne à la rédaction de documents méthodologiques (protocole de rédaction de fiches, arbres des domaines, méthodologie des recherches ponctuelles ct thématiques, etc.)?

- $\operatorname{rmp}: 6 \%$

Quel pourcentage de votre temps consacrez-vous en moyenne à la formation (stagiaires, nouveaux terminologues, clients, traducteurs, initiation aux banques, etc.) ?

- $\operatorname{rmp}: 10 \%$

Combien de temps consacrez-vous en moyenne par an à la participation à des colloques ?

- $\operatorname{rmp}: 4$ jours

Combien de temps consacrez-yous en moyenne par an à des missions de terminologie ou de documentation?

- $\operatorname{rmp}: 4$ jours

Combien de temps consacrez-vous en moyenne par année, durant les heures de travail, à des activités professionnelles du type réunions, organisation de colloques, etc. ?

a $\operatorname{rmp}: 5$ jours.

\section{Annexe 2}

\section{FICHE CTN / IA}

FRA 65 vedette

GRA 3 partie du discours

SOU $69 \quad$ source : auteur, type de support, lieu et date

LEN 3 code LENOCH (système de classification de la Commission des Communautés européennes)

DOM $30 \quad$ domaine

SDO 69 sous-domaines ou domaines d'application (ensembles thématiques correspondant eux-mêmes à des notions)

DEF 254 définition ou énoncé définitoire

CON 254 contexte; complément d'information textuel

NOT $200 \quad$ notes; renseignements de type encyclopédique

ENG 65 équivalents, synonymes ou abréviations anglais

SYN 65 synonymes et équivalents français (et abréviations et variantes orthographiques)

STA 65 standard, norme (avec mention de l'organisme, de l'autorité de normalisation: ISO, AFNOR, OLF, BTQ...)

COM $50 \quad$ combinaison; syntagme dont le terme est un constituant immédiat typique : verbe support, préposition, déterminant...

ACT 65 action typique, prédicat de fonction (ce à quoi sert un objet, par exemple)

OBJ 65 objet connexe; notion à laquelle les experts associent le plus spontanément celle qui est décrite

SOR $50 \quad$ sorte de ; genre / espèce ; concept générique immédiatement supérieur dans une classification

ANA $50 \quad$ anaphore : hyperonymie, répétition, nominalisation...

PAR $50 \quad$ partie de ; le tout dont la notion considérée est une partie (composant)

$\mathrm{CO} 140$ élément formant; premier élément de l'ordre syntagmatique (décomposition binaire); constituant 1

$\mathrm{CO} 2 \quad 40 \quad$ constituant 2

RED 10 nom du centre + nom du rédacteur

ANN 4 année de l'énoncé

MAJ 4 anné de mise à jour 
PRE 4 première datation connue

REP 2 repère; coefficient de fiabilité

FAM 69 famille; renvois, termes reliés
GABRIEL OTMAN Centre de terminologie et de néologie, Paris, France 